\title{
Sensitivity-enhanced three-dimensional and carbon-detected two-dimensional NMR of proteins using hyperpolarized water
}

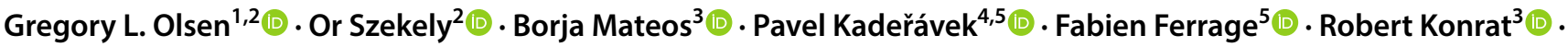

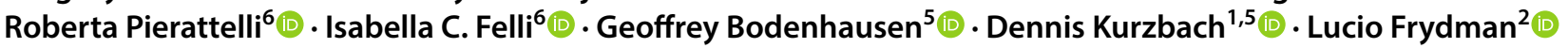

Received: 9 September 2019 / Accepted: 27 January 2020 / Published online: 10 February 2020

(c) The Author(s) 2020

\begin{abstract}
Signal enhancements of up to two orders of magnitude in protein NMR can be achieved by employing HDO as a vector to introduce hyperpolarization into folded or intrinsically disordered proteins. In this approach, hyperpolarized HDO produced by dissolution-dynamic nuclear polarization (D-DNP) is mixed with a protein solution waiting in a high-field NMR spectrometer, whereupon amide proton exchange and nuclear Overhauser effects (NOE) transfer hyperpolarization to the protein and enable acquisition of a signal-enhanced high-resolution spectrum. To date, the use of this strategy has been limited to $1 \mathrm{D}$ and ${ }^{1} \mathrm{H}_{-}{ }^{15} \mathrm{~N} 2 \mathrm{D}$ correlation experiments. Here we introduce $2 \mathrm{D}{ }^{13} \mathrm{C}$-detected D-DNP, to reduce exchange-induced broadening and other relaxation penalties that can adversely affect proton-detected D-DNP experiments. We also introduce hyperpolarized 3D spectroscopy, opening the possibility of D-DNP studies of larger proteins and IDPs, where assignment and residue-specific investigation may be impeded by spectral crowding. The signal enhancements obtained depend in particular on the rates of chemical and magnetic exchange of the observed residues, thus resulting in non-uniform 'hyperpolarizationselective' signal enhancements. The resulting spectral sparsity, however, makes it possible to resolve and monitor individual amino acids in IDPs of over 200 residues at acquisition times of just over a minute. We apply the proposed experiments to two model systems: the compactly folded protein ubiquitin, and the intrinsically disordered protein (IDP) osteopontin (OPN).
\end{abstract}

Keywords Hyperpolarization · Dissolution-dynamic nuclear polarization (D-DNP) $\cdot$ Direct ${ }^{13} \mathrm{C}$ detection $\cdot 3 \mathrm{D}$ NMR $\cdot$ Nonuniform sampling $\cdot$ BEST-HNCO

Electronic supplementary material The online version of this article (https://doi.org/10.1007/s10858-020-00301-5) contains supplementary material, which is available to authorized users.

Gregory L. Olsen

Gregory.Olsen@univie.ac.at

$\triangle$ Dennis Kurzbach

Dennis.Kurzbach@univie.ac.at

1 Faculty of Chemistry, Institute for Biological Chemistry, University of Vienna, Währinger Straße 38, 1090 Vienna, Austria

2 Department of Chemical and Biological Physics, Weizmann Institute of Science, Rehovot, Israel

3 Department of Structural and Computational Biology, University of Vienna, Vienna BioCenter 5, 1030 Vienna, Austria

\section{Introduction}

Nuclear magnetic resonance (NMR) spectroscopy is one of the principal tools for the investigation of the structural dynamics and structure-function relationships of proteins in solution (Theillet et al. 2016; Reichheld et al. 2017; Gil et al.

4 CEITEC-Central European Institute of Technology, Masaryk University, Kamenice 5, 62500 Brno, Czech Republic

5 Laboratoire des biomolécules, LBM, Département de chimie, École normale supérieure, PSL University, Sorbonne Université, CNRS, 75005 Paris, France

6 Magnetic Resonance Center and Department of Chemistry Ugo Schiff, University of Florence, Via L. Sacconi 6, 50019 Sesto Fiorentino, FI, Italy 
2013; Yuwen and Skrynnikov 2014). A significant limitation of the method, however, is its low sensitivity, which can necessitate extensive signal-averaging. The acquisition of protein spectra thus often extends to hours or days, which can be prohibitive for the study of systems which are evolving rapidly or have limited stability in solution. We have recently explored the use of hyperpolarized water (Lipso et al. 2017) to boost signals in protein spectra. Hyperpolarized HDO produced using dissolution-dynamic nuclear polarization (D-DNP) can serve as a polarization reservoir, enhancing the ${ }^{1} \mathrm{H}$ signals of sites undergoing chemical- or polarization exchange with the hyperpolarized solvent. This approach, which has been referred to as HYPEX (Kadeřávek et al. 2018) or HyperW (Szekely et al. 2018), is applicable to a broad range of aqueous systems without requiring any special properties or chemical modification of the molecules under study, and makes it possible to achieve over 100-fold signal enhancements in spectra of folded or intrinsically disordered proteins (Kadeřávek et al. 2018; Szekely et al. 2018; Kurzbach et. al. 2017; Olsen et al. 2016; Kim et al. 2017; Ragavan et al. 2017; Wang and Hilty 2019; Doll et al. 2012; Viennet et al. 2016).

In the present study, we expand this approach to include three-dimensional (3D) and ${ }^{13} \mathrm{C}$-detected $2 \mathrm{D}$ experiments (Gil et al. 2013), which we demonstrate using two representative protein systems: the compactly folded model protein ubiquitin (Vijay-Kumar and Bugg 1987; Hamilton et al. 2000), and the intrinsically disordered protein (IDP) osteopontin (OPN), and its complex with its ligand heparin (Kurzbach et al. 2013; Rodrigues et al. 2007). Using the proposed experiments, we obtain hyperpolarized 'fingerprints' of the two proteins, and are also able to detect a minor OPN population that would likely have remained undetected by conventional thermal equilibrium NMR spectroscopy.

\section{Results and discussion}

The hyperpolarized HDO-based buffers that are at the heart of this experimental strategy were produced using D-DNP systems operating at $\sim 1.2 \mathrm{~K}$ and magnetic fields of either 3.35 T (Oxford HyperSense ${ }^{\mathrm{TM}}$ ) or 6.7 T (Bruker BioSpin). The general procedure is as follows (for details see the Experimental section): (i) Hyperpolarization of a frozen $\mathrm{H}_{2} \mathrm{O}$ solution containing a paramagnetic polarization agent and a cryo-protectant is achieved by means of off-resonance microwave irradiation. (ii) After building up the polarization for 2-3 h, the hyperpolarized $\mathrm{H}_{2} \mathrm{O}$ is dissolved with a burst of superheated neat or buffered $\mathrm{D}_{2} \mathrm{O}$ to produce a mixture containing 1-4\% HDO (depending on the system and experimental parameters), which is then injected in 1-3 s into an NMR tube waiting in a high-field detection spectrometer, where it mixes in-situ with the target protein solution. (iii) Chemical and 'magnetic' exchange processes (transient Overhauser effects) between hyperpolarized HDO and the protein transfer ${ }^{1} \mathrm{H}$-hyperpolarization to the protein, thereby selectively enhancing NMR signals of residues with favorable solvent interactions (Kadeřávek et al. 2018; Szekely et al. 2018; Kurzbach et al. 2017; Olsen et al. 2016; Kim et al. 2017).

Signal intensities for all experiments are tabulated in the Supplementary Information. In most cases, even after extensive signal averaging, the signals in thermal equilibrium spectra for the D-DNP samples were too weak for a reliable determination of the enhancement factor ' $\varepsilon$ ' (often defined as the ratio between per-scan signal amplitudes in the hyperpolarized spectrum and the corresponding spectrum acquired after return of the system to thermal equilibrium). Therefore, to quantify the signal intensities obtained with hyperpolarized HDO, we instead report signal-to-noise ratios (SNR) for the hyperpolarized spectra. (As an approximate lower bound for $\varepsilon$, one can thus assume that $\varepsilon \geq \operatorname{SNR}$ for instances where the corresponding reference signal remains below the limit of detection).

\section{Hyperpolarized high-resolution ${ }^{13} \mathrm{C}$-detected 2D NMR}

In previous work, we demonstrated how solvent hyperpolarization can be combined with rapid $2 \mathrm{D}^{1} \mathrm{H}^{-}{ }^{15} \mathrm{~N}$ HMQC spectroscopy (Schanda et al. 2005) of folded proteins and small IDPs (Kadeřávek et al. 2018; Szekely et al. 2018; Kurzbach et al. 2017; Olsen et al. 2016; Kim et al. 2017). However, analysis of proton-detected hyperpolarized 2D spectra of larger proteins or IDPs is sometimes challenging, as signal overlap and broadening due to exchange, radiation damping, and paramagnetic relaxation may hinder spectral analysis. To address these limitations, we expand our approach here to include ${ }^{13} \mathrm{C}$-detected ${ }^{13} \mathrm{C}-{ }^{15} \mathrm{~N}$ correlation experiments $\left(\mathrm{H}^{\mathrm{N}}-\mathrm{CON}\right.$, see pulse sequence in Fig. S1) (Gil et al. 2013; Bertini et al. 2011). In these experiments, chemical and magnetic exchange transfer proton hyperpolarization from HDO to protein backbone amide (and side-chain) sites. The amide hyperpolarization is then transferred by selective INEPT to the neighboring ${ }^{15} \mathrm{~N}$ nuclei, and, after an ${ }^{15} \mathrm{~N}$ evolution period, transferred onward to the adjacent ${ }^{13} \mathrm{CO}$ spins for detection (Gil et al. 2013). The amide proton polarization is thus continuously replenished in successive scans by the hyperpolarized HDO pool, while ${ }^{1} \mathrm{H}$-detection is avoided.

Direct ${ }^{13} \mathrm{C}$ detection offers the added advantage that the chemical shift dispersion in the ${ }^{13} \mathrm{C}$ dimension is much larger compared to ${ }^{1} \mathrm{H}$, while at the same time the penalties associated with ${ }^{1} \mathrm{H}$ detection are reduced. 
Hyperpolarized $\mathrm{H}^{\mathrm{N}}-\mathrm{CON}\left(98 \% \mathrm{D}_{2} \mathrm{O}\right) ; 69 \mathrm{~s} \quad \mathrm{NS}=2 ; 16$ compl. $t_{1}$ inc. Conventional $\mathrm{H}^{\mathrm{N}}-\mathrm{CON}\left(10 \% \mathrm{D}_{2} \mathrm{O}\right) ; 186 \mathrm{~s} \quad \mathrm{NS}=2 ; 20$ compl. $t_{1}$ inc.

(a)

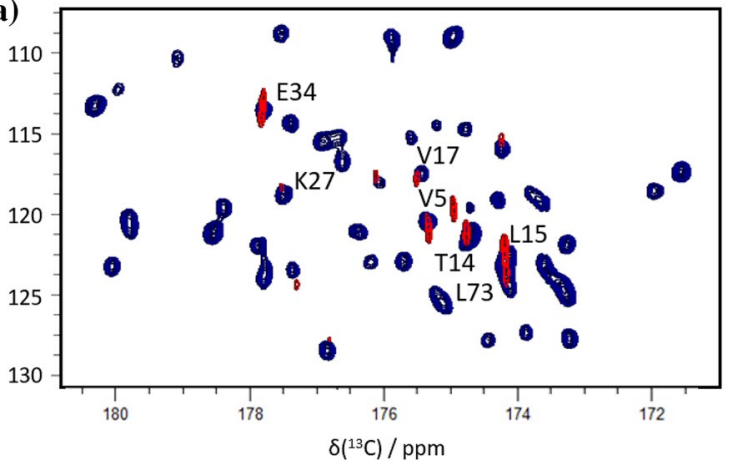

(b)

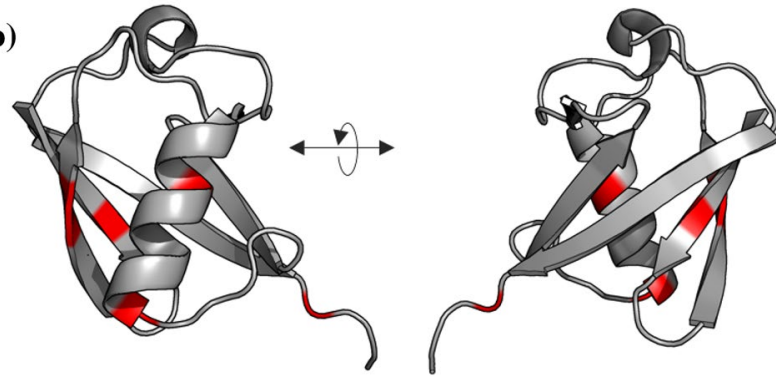

Fig. 1 a Hyperpolarized $\mathrm{H}^{\mathrm{N}}-\mathrm{CON}$ correlation spectrum of ubiquitin in $98 \%$ deuterated buffer (red signals), superimposed on the corresponding thermal equilibrium spectrum obtainedin $10 \%$ deuterated buffer (blue peaks). The assignments denote residue $\{i\}$, for each $\{i$, $i-1\}{ }^{15} \mathrm{~N}-{ }^{13} \mathrm{C}$ cross peak. b Ubiquitin crystal structure (PDB code 1ubq), with the subset of residues detected in experiments with hyperpolarized HDO highlighted in red. These consist primarily of residues undergoing rapid solvent exchange, but also include several cases where the enhanced polarization has migrated to adjacent core residues. (Signal intensities are tabulated in Supplementary Table S1.)

\section{Application to a folded protein: Ubiquitin}

A hyperpolarized $\mathrm{H}^{\mathrm{N}}-\mathrm{CON}$ spectrum obtained for ubiquitin via this strategy is shown in Fig. 1a (red), overlaid with a conventional $\mathrm{H}^{\mathrm{N}}-\mathrm{CON}$ spectrum of ubiquitin obtained at thermal equilibrium in a $90: 10 \% \mathrm{H}_{2} \mathrm{O}: \mathrm{D}_{2} \mathrm{O}$ phosphate buffered solution (blue). The hyperpolarized spectrum was acquired in $69 \mathrm{~s}$ at $37^{\circ} \mathrm{C}$. Both spectra were obtained in a magnetic field of $14.1 \mathrm{~T}(600 \mathrm{MHz})$.

Only if chemical and magnetic exchange from HDO is sufficiently fast will enhanced signals be observed (Kadeřávek et al. 2018; Szekely et al. 2018; Kurzbach et al. 2017; Olsen et al. 2016; Kim et al. 2017; Nucci et al. 2011; Otting 1997; Modig et al. 2004). In particular, the signal enhancement for an amide site should be increased by faster exchange rates on the one hand, as replenishment of hyperpolarized amide protons between successive scans is improved, while on the other hand the improvement will be attenuated by losses due to proton exchange and relaxation during evolution delays and detection. Thus, as expected, the hyperpolarized spectrum contains a reduced subset of the signals seen in the corresponding conventional spectrum. Figure $1 \mathrm{~b}$ maps the observed signals onto the crystal structure of ubiquitin, revealing that some residues in the hydrophobic core are also detected, indicating that hyperpolarization may in some cases be transferred from the solventexposed surface to the protein's inner core.

The set of peaks observed depends on many variables, in particular on the overall SNR characteristic of the experiment in question, the delay between successive detections, and as noted above, the hyperpolarization transfer efficiency through proton exchange, nuclear Overhauser effects (NOE), or exchange-relayed NOE. While the latter three polarization transfer pathways do not critically depend on the pulse sequence or nucleus used for detection, the SNR of course does. However, if the underlying HYPEX/HyperW exchange and NOE polarization transfer mechanisms are operating as in our earlier proton-detected work (Kadeřávek et al. 2018), one should anticipate a qualitative agreement between the set of residues observed in the hyperpolarized protondetected ${ }^{1} \mathrm{H} /{ }^{15} \mathrm{~N}$ HMQC spectrum reported there and in the ${ }^{13} \mathrm{C}$-detected spectrum we obtain here. And indeed, the $\mathrm{H}^{\mathrm{N}}$-CON results confirm these expectations, as all peaks observed with $\mathrm{H}^{\mathrm{N}}-\mathrm{CON}$ detection were also observed in the hyperpolarized HMQC. Note that in the $\mathrm{H}^{\mathrm{N}}-\mathrm{CON}$, we do not see all of the peaks seen in the HMQC spectrum; we see instead only the set of peaks which showed the highest SNR in the proton-detected experiment. The reduction in the number of detected residues is due to several factors, such as the additional coherence transfer steps in the $\mathrm{H}^{\mathrm{N}}-\mathrm{CON}$ experiments and the intrinsically lower sensitivity of ${ }^{13} \mathrm{C}$ detection.

Moreover, as seen in Fig. 1a, despite the rapid injections and brief experimental durations used, the linewidths in the directly-detected dimension of the hyperpolarized $\mathrm{H}^{\mathrm{N}}-\mathrm{CON}$ $(10-15 \mathrm{~Hz})$ are comparable to or narrower than those in its conventional counterpart, and approximately half of those obtained previously using ${ }^{1} \mathrm{H}$-detection $(25-30 \mathrm{~Hz}$ in hyperpolarized ${ }^{1} \mathrm{H} /{ }^{15} \mathrm{~N}$ HMQC) (Kadeřávek et al. 2018), indicating that ${ }^{13} \mathrm{C}$-detection reduces radiation damping and/ or decoherence due to rapid proton exchange and paramagnetic relaxation - factors that limited resolution in earlier proton-detected hyperpolarized studies of protein systems (Kurzbach et al. 2017).

\section{Application to an IDP: Osteopontin and its complex with heparin}

Figure $2 \mathrm{a}$ displays a hyperpolarized ${ }^{13} \mathrm{C}-{ }^{15} \mathrm{~N}$ spectrum of a 220-residue truncation mutant of OPN. Figure $2 b$ shows a thermal equilibrium reference spectrum collected in a $10 \%$ deuterated buffer, and Fig. 2c shows a comparison of 

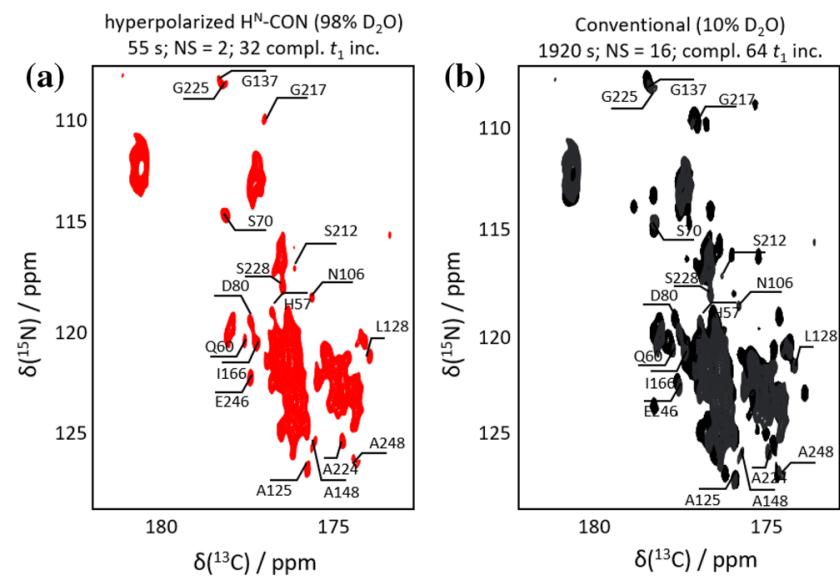

Fig. 2 a Hyperpolarized $\mathrm{H}^{\mathrm{N}}$-CON correlation spectrum of osteopontin detected in $55 \mathrm{~s}$ using hyperpolarized HDO, in ca. 98\% deuterated buffer, with assignments of a few well-resolved residues. b conventional reference spectrum acquired in $10 \%$ deuterated buffer. c Overlay of the hyperpolarized spectrum (red) and the reference spectrum

the hyperpolarized and reference spectra. The hyperpolarized spectrum was obtained in $55 \mathrm{~s}$ at $37^{\circ} \mathrm{C}$ and $\mathrm{pH} 7.4$ at $14.1 \mathrm{~T}\left(600 \mathrm{MHz}\right.$ for $\left.{ }^{1} \mathrm{H}\right)$. As for ubiquitin, the hyperpolarized and conventional $\mathrm{H}^{\mathrm{N}}$-CON spectra show nearly identical linewidths. In contrast to the folded ubiquitin, however, a markedly larger fraction of the peaks observed in the conventional spectrum of OPN are also present in the hyperpolarized spectrum. This is not surprising, given the greater solvent exposure expected for an IDP, which would be consistent with particularly efficient chemical exchange and polarization transfer.

A strikingly different spectral response is observed when OPN is monitored after mixing with its ligand heparin (Clemente 2016). The heparin interaction site spans OPN residues 100 to 180 . Upon binding of the large $17 \mathrm{kDa}$ ligand, much of the IDP is in direct contact with heparin, and thus likely becomes somewhat shielded from the solvent. Indeed, when probed by hyperpolarized $\mathrm{H}^{\mathrm{N}}$-CON after addition of heparin, the number of detected OPN peaks drops significantly, and the subset of observed residues changes markedly as well (Fig. 2d).

Due to signal overlap, numerous peaks observed in both the conventional and hyperpolarized spectra of OPN cannot be assigned with $2 \mathrm{D} \mathrm{HMQC}$ or $\mathrm{H}^{\mathrm{N}}-\mathrm{CON}$ data alone, making it difficult to determine the true spectral 'binding footprint' for this system. However, the fact that the pronounced drop in observed signals is not restricted to the binding site suggests that screening by the ligand alone is not the only determinant of changes in polarization transfer efficiency in the complex. Complex formation would also be expected to affect solvent exposure and polarization transfer of newly-sequestered residues substantially, perhaps
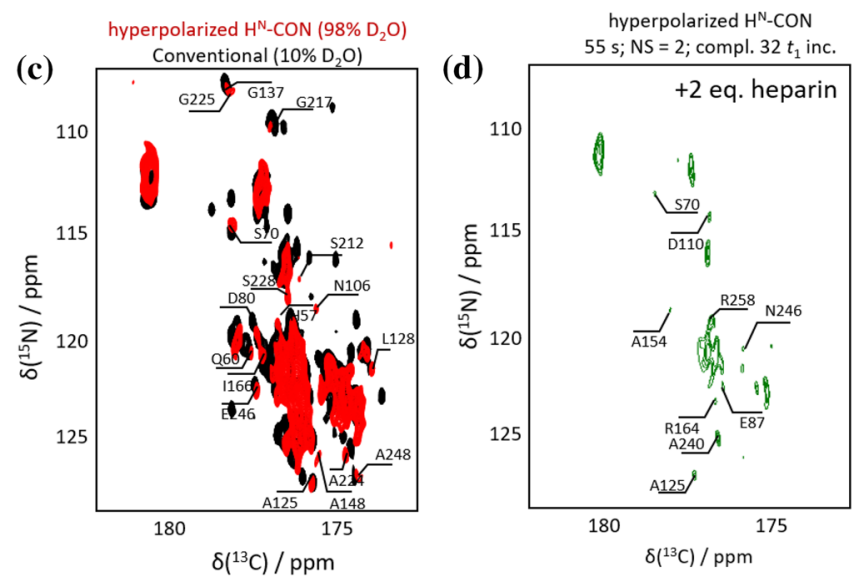

(black). d Hyperpolarized $\mathrm{H}^{\mathrm{N}}$-CON correlation spectrum of OPN in the presence of 2 equivalents of heparin, with assignments of some well-resolved residues. (Signal intensities are tabulated in Supplementary Tables S2 and S3.)

resulting in a sparse enhancement pattern not unlike that seen for ubiquitin.

\section{Hyperpolarized three-dimensional NMR}

While the improvements in resolution provided by the hyperpolarized $\mathrm{H}^{\mathrm{N}}$-CON relative to hyperpolarized ${ }^{1} \mathrm{H}_{-}{ }^{15} \mathrm{~N}$ HMQC experiments can be substantial, particularly for samples such as OPN (Mateos et al. 2019), in many instances 2D NMR cannot reduce signal overlap sufficiently to permit residue-specific analysis. To further reduce spectral crowding and to widen the scope of our approach, we therefore implemented hyperpolarized $3 \mathrm{D}{ }^{1} \mathrm{H}-{ }^{13} \mathrm{C}-{ }^{15} \mathrm{~N}$ correlation experiments-in particular, the BEST-HNCO experiment shown in Fig. S2 (Lescop et al. 2007).

\section{Methodological considerations}

Given a final solvent deuteration level of 96-98\% after dissolution and mixing, typical proton hyperpolarization lifetimes in these experiments are less than $2 \min \left(T_{1}\left({ }^{1} \mathrm{H}\right) \approx 20 \mathrm{~s}\right)$. To permit detection of two indirectly encoded dimensions under this time constraint, the use of non-uniform sampling (NUS) (Zawadzka-Kazimierczuk et al. 2012; Mayzel et al. 2014) is mandatory. In the present case, after empirical optimization, $9 \%$ NUS was chosen to provide a compromise between spectral resolution along $\omega_{1}\left({ }^{13} \mathrm{C}\right)$ and $\omega_{2}\left({ }^{15} \mathrm{~N}\right)$, while allowing us to complete the $3 \mathrm{D}$ acquisitions within the time restriction of the HDO polarization lifetime. Poisson gap sampling (Hyberts et al. 2012) was used to generate NUS schedules, and a total of 96 FIDs were recorded in 
65-75 s for a nominal digital resolution of $44 \times 89 \times 352 \mathrm{~Hz}$ in $\omega_{3}, \omega_{2}$ and $\omega_{1}$ respectively. The hyperpolarized HNCO spectra presented here were acquired in $65 \mathrm{~s}$ (OPN + Heparin) or $75 \mathrm{~s}$ (ubiquitin, OPN) at $18.8 \mathrm{~T}$, in $96 \%$ deuterated buffer, at $\mathrm{pH} 7.4$ and $37^{\circ} \mathrm{C}$.

The full 3D spectra were reconstructed using hmsIST (Hyberts et al. 2012) in NMRPipe (Delaglio et al. 1995), and all spectra were cross-checked against conventional thermal equilibrium spectra to identify potential spectral reconstruction artifacts. While many alternative NUS strategies are available (Kazimierczuk et al. 2009, 2010), the HMS implementations of sampling and reconstruction were chosen for the proof-of-concept experiments described here, as they are readily available to the NMR community and robust in our hands (https://gwagner.med.harvard.edu/intranet/hmsIST/).

\section{Ubiquitin}

As for the selectively hyperpolarized $\mathrm{H}^{\mathrm{N}}-\mathrm{CON}$ experiments described above, we first tested the hyperpolarized HNCO using ubiquitin as a representative folded protein. Figure $3 \mathrm{a}$ displays the projection of the ${ }^{1} \mathrm{H}-{ }^{15} \mathrm{~N}$ plane of a hyperpolarized 3D HNCO spectrum collected in just over one minute overlaid onto a conventional ${ }^{1} \mathrm{H}_{-}{ }^{15} \mathrm{~N} 2 \mathrm{D}$ HSQC of ubiquitin detected in $2 \mathrm{~h}$ (in 90:10 $\mathrm{H}_{2} \mathrm{O}: \mathrm{D}_{2} \mathrm{O}$, at $\mathrm{pH} 7.4$ and $37{ }^{\circ} \mathrm{C}$ ). Representative planes of the hyperpolarized HNCO spectrum are shown in Fig. 3b. While the conventional 2D reference spectrum has better resolution than its hyperpolarized $3 \mathrm{D}$ counterpart, as expected, the subset of peaks obtained in the hyperpolarized HNCO spectrum are in good agreement with their counterparts in the conventional 2D spectrum, and the linewidths in the NUS 3D are near the nominal resolution expected for an equivalent 'fully-sampled' $75 \mathrm{~s}$ acquisition ( ${ }^{15} \mathrm{~N}$ nominal resolution: $89 \mathrm{~Hz}(\sim 1.1 \mathrm{ppm}) ;{ }^{15} \mathrm{~N}$ observed resolution: 1-2 ppm). Figure $3 \mathrm{c}$ maps the detected residues (in red) onto the ubiquitin crystal structure. This set of detected signals corresponds to the one obtained by the hyperpolarized $\mathrm{H}^{\mathrm{N}}-\mathrm{CON}$ (Fig. 1b), as all peaks observed in the $\mathrm{H}^{\mathrm{N}}-\mathrm{CON}$ are also observed in the HNCO. Notably, as was seen previously in the hyperpolarized HMQC of ubiquitin (Kadeřávek et al. 2018), significant enhancements are observed not only for solvent-exposed surface residues, but also for several residues in the protein core.

\section{Osteopontin and its ligand heparin}

Figure $4 \mathrm{a}, \mathrm{b}$ shows representative planes of the hyperpolarized HNCO spectra of the heparin-free and -bound states of
Fig. 3 a Overlay of a conventional ${ }^{1} \mathrm{H}-{ }^{15} \mathrm{~N}$ HSQC spectrum of ubiquitin obtained in $2 \mathrm{~h}$ (blue) and the projection onto the ${ }^{1} \mathrm{H}-{ }^{15} \mathrm{~N}$ plane of a selectively hyperpolarized 3D HNCO spectrum of ubiquitin obtained in 75 s using NUS (red). Assignments for ubiquitin at $\mathrm{pH} 7.4$ were based upon $\mathrm{pH}$ titration experiments (see Fig. S10). Asterisks denote possible reconstruction artifacts. b Planes representative of the selectively hyperpolarized HNCO. c Selectively hyperpolarized residues (red) mapped onto the crystal structure (PDB code 1ubq) of ubiquitin. (Signal intensities are tabulated in Supplementary Table S4.)
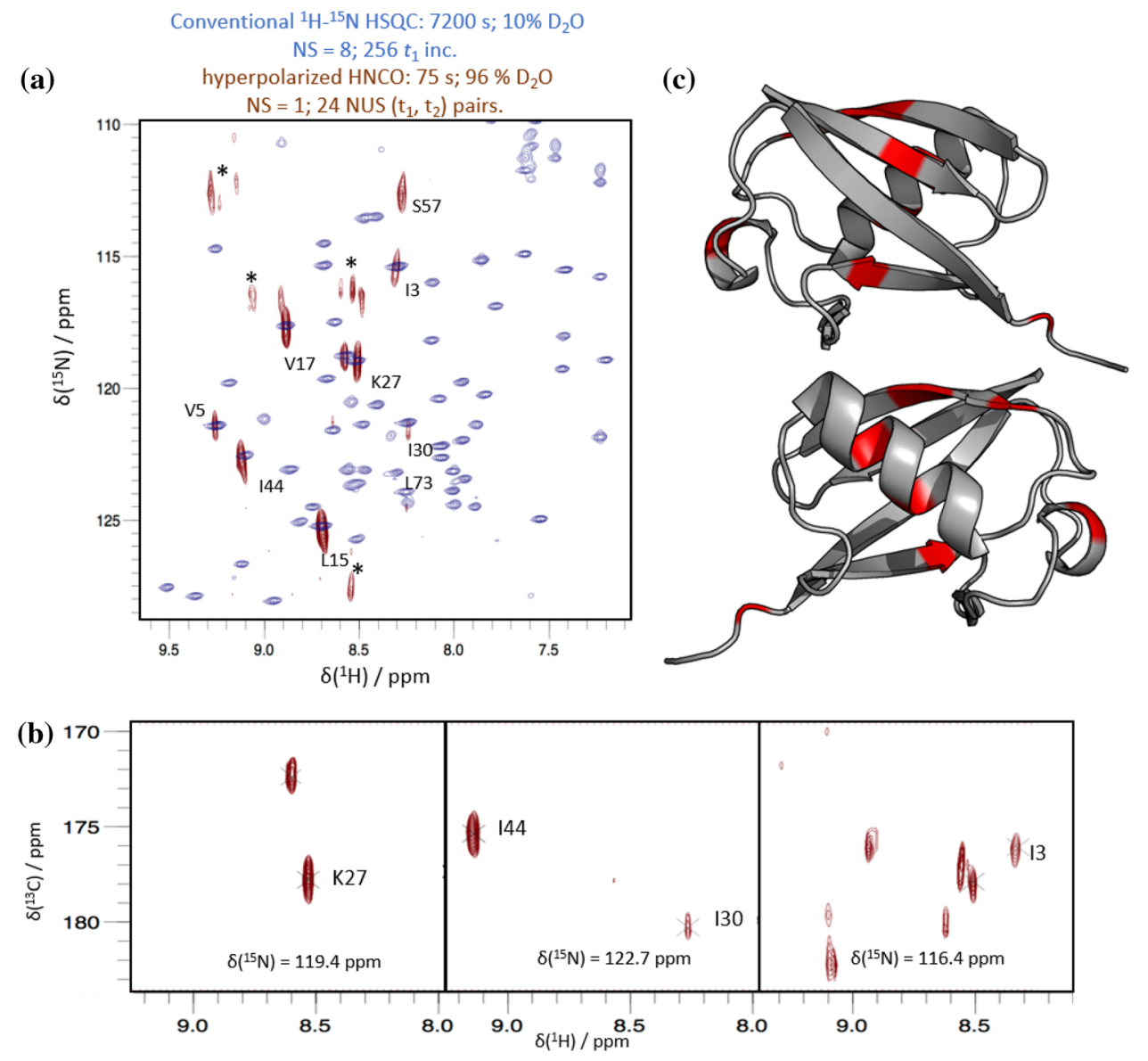

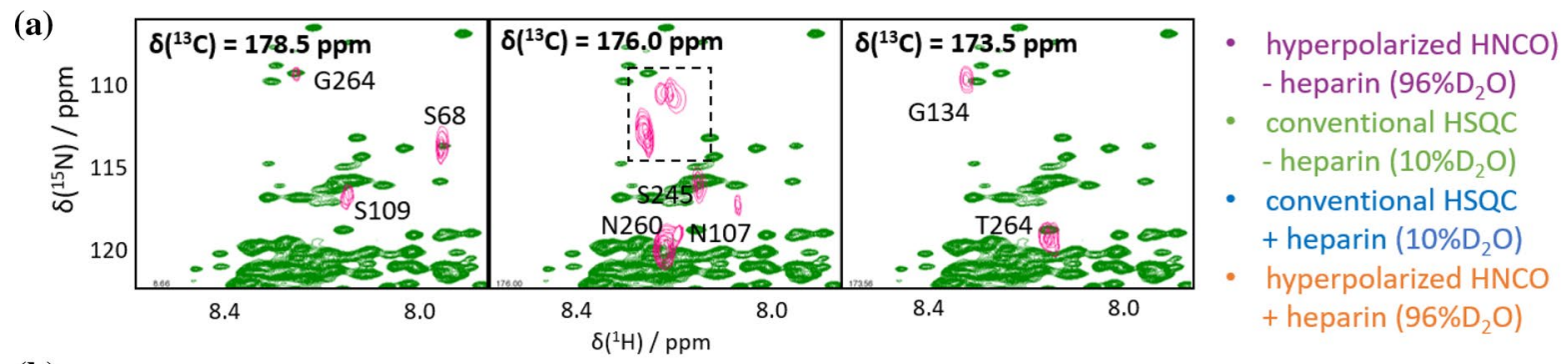

(b)
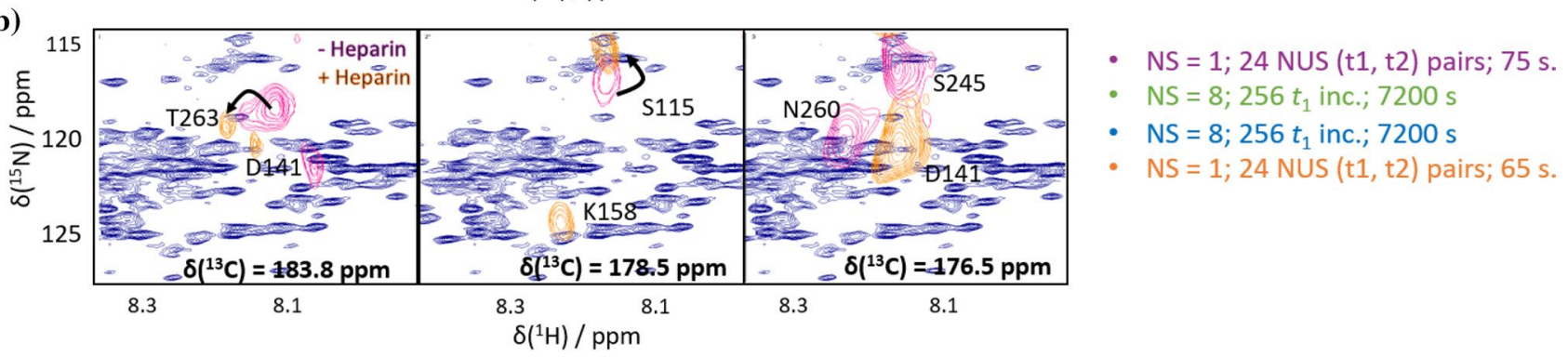
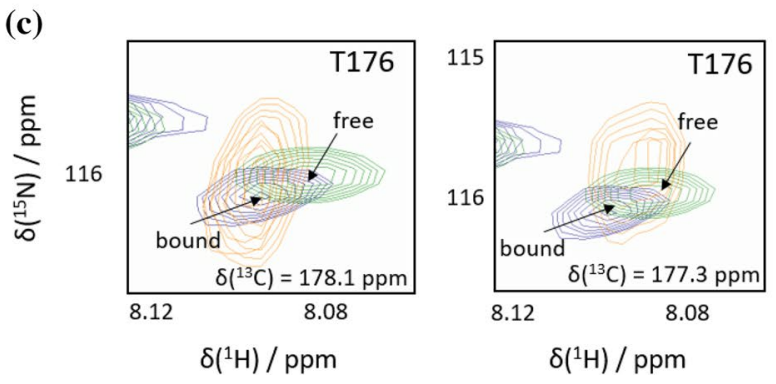

Fig. 4 Detection of heparin binding to OPN: a Overlay of a conventional thermal equilibrium $2 \mathrm{D}{ }^{1} \mathrm{H}_{-}{ }^{15} \mathrm{~N}$ HSQC spectrum of free OPN in $10 \%$ deuterated buffer (green) with three planes from a hyperpolarized 3D NUS HNCO in 96\% deuterated buffer (magenta). The dashed box indicates signals of arginine side chains that are folded in both the ${ }^{13} \mathrm{C}$ - and the ${ }^{15} \mathrm{~N}$ dimension. b Overlay of the conventional $2 \mathrm{D}{ }^{1} \mathrm{H}$ ${ }^{15} \mathrm{~N}$ HSQC spectrum of OPN in the presence of 2 equivalents of heparin (blue) and three hyperpolarized 3D NUS HNCO planes (orange: with heparin; magenta: without heparin). c Superpositions of three zoomed planes from the hyperpolarized HNCO spectrum in the presence of heparin (orange), and conventional ${ }^{1} \mathrm{H}^{-15} \mathrm{~N}$ HSQCs of OPN in the presence (blue) and absence (green) of heparin. Both ligandfree and ligand-bound states are detectable in the HNCO spectrum for residue T176, which is located in the binding site and experiences a chemical shift upon heparin binding. The signal of the heparin-bound

OPN (magenta and orange peaks, respectively), overlaid on the corresponding conventional thermal equilibrium HSQC spectra (green and blue, respectively). Figure $4 \mathrm{c}$ illustrates the residue-selectivity of the hyperpolarization in the presence and absence of heparin. The signal overlap in conventional 2D spectra of this system would make a transfer of assignments and spectral analysis particularly challenging (see also the Supporting Information). Here, extension of hyperpolarized experiments to encompass a third spectral dimension provides a significant improvement in resolution, and is also able to highlight several features of the OPNheparin interaction.

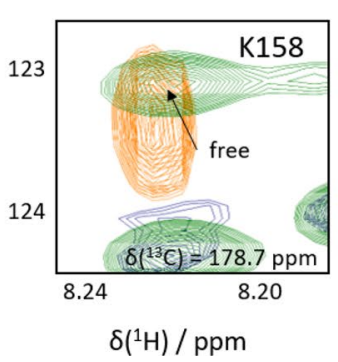

(d)

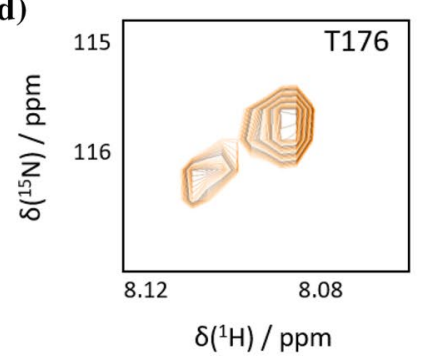

form of OPN can be seen in the $\delta\left({ }^{13} \mathrm{C}\right)=178.1 \mathrm{ppm}$ HNCO plane (left), where the bound-form peak (in yellow) coincides with that of the heparin-bound reference (blue), while the heparin-free form is observed in the $\delta\left({ }^{13} \mathrm{C}\right)=177.3 \mathrm{ppm} \mathrm{HNCO}$ plane (center), where the signal now coincides with the heparin-free reference (green). For residue K158 (also located in the binding site; right), only the signal of the free form is observed in the presence of heparin, whereas in the bound form this residue is broadened beyond detection in both conventional and hyperpolarized spectra. (Signal intensities are tabulated in Supplementary Tables S5 and S6.) d Superposition of the zoomed ${ }^{1} \mathrm{H}^{15} \mathrm{~N}$ planes of the $\mathrm{HNCO}$ shown at left and center in (c) $\left(\right.$ at $\delta\left({ }^{13} \mathrm{C}\right)=177.3 \mathrm{ppm}$ and $\left.\delta\left({ }^{13} \mathrm{C}\right)=178.1 \mathrm{ppm}\right)$ ), at different cut-off levels showing the two distinct peaks which can be discerned for residue $\mathrm{T} 176$ in the 3D experiment

Notably, in the presence of 2 equivalents of the ligand, traces of a second small population were seen, which we attribute to the unbound IDP. This could not be observed in conventionally detected spectra of the complex. This is illustrated in Fig. 4c, d, where spectral signatures of both the free and bound forms of OPN are detected for residue T176, while in the corresponding conventional spectrum, only the bound form is seen. If we assume that free OPN is not screened by the ligand, and thus more readily enhanced, solvent-based hyperpolarization would be expected to be particularly effective in highlighting the small population of free OPN even in the presence of a much larger population 
of the bound state. For residue K158 this effect is indeed observed, as only the free form is seen in the presence of heparin. While the $\mathrm{pH}$ and temperature used here (7.4, $37{ }^{\circ} \mathrm{C}$ ) differ from those used in previous studies of this system, which may affect the visibility of certain residues, in any case the ability to detect such small populations should prove valuable for the characterization of sparsely-populated states.

Due to substantial overlap, the resolution in hyperpolarized 1D or 2D spectroscopy would not be sufficient to distinguish these two signals (see Fig. S9 in the Supporting Information for representative $1 \mathrm{D}$ projections). In the 3D experiments, however, these peaks are well isolated from other resonances and thus could be unambiguously assigned. As can be seen in Fig. $4 \mathrm{c}$, the ${ }^{15} \mathrm{~N}$ chemical shifts of the two T176 signals are in good but not perfect agreement with those observed for the two peaks in this region of the static reference spectra. While we cannot exclude the possibility that alternative OPN conformations giving a similar but slightly shifted spectral signature could be present in the sample, the presence of distinct T176 signals in the 3D spectrum is consistent with the presence of both a bound and an unbound OPN species following heparin addition. Thus, by combining the improved sensitivity provided by hyperpolarized water with the improved resolution afforded by $3 \mathrm{D}$ detection, signatures of even small populations of minor states can be discerned.

\section{Conclusions}

The present study demonstrates how, by combining the use of hyperpolarized water with rapid $3 \mathrm{D}$ - and ${ }^{13} \mathrm{C}$-detected $2 \mathrm{D}$ experiments, we are able to take advantage of the enhanced sensitivity available in earlier hyperpolarized $1 \mathrm{D}$ and $2 \mathrm{D}$ ${ }^{1} \mathrm{H}_{-}{ }^{15} \mathrm{~N}$ HMQC experiments while providing the improved resolution needed to extend the method to larger proteins and IDPs.

Hyperpolarized HDO-based experiments such as those introduced here typically produce spectra showing nonuniform 'hyperpolarization-selective' signal enhancements. Many factors need to be considered when trying to predict the likelihood that the intensity of a given residue will be boosted by means of hyperpolarized solvents, most importantly the proton exchange rate, but also the contributions of direct- or exchange-relayed NOE (Kadeřávek et al. 2018). As noted above, the improvement in signal intensity for an amide site is increased by faster exchange rates on the one hand, as replenishment of hyperpolarized amide protons between successive scans is improved, while on the other hand the improvement is attenuated by losses due to proton exchange and relaxation during transfer, evolution, and detection. Moreover, the efficiency of the hyperpolarization also depends on the details of local solvation, on the ionic strength, and on the polarization and relaxation rate of the HDO, so that reliable predictions are challenging.

However, it is already clear that the use of hyperpolarized water to boost protein signals not only provides a more sensitive detection, but also achieves a 'hyperpolarizationselective labeling', carrying valuable insight about the rates of chemical and magnetic (NOE) polarization exchange between solvent and protein.

It should also be noted that the experiments presented here suffer a substantial SNR penalty as a result of the dilution of the hyperpolarized water during the dissolution process: in contrast to the $90 \% \mathrm{H}_{2} \mathrm{O}$ buffers commonly used in standard NMR measurements, the final $\mathrm{H}_{2} \mathrm{O}$ fractions available to populate the protein sites are typically reduced to $2-4 \%$ after mixing of the hyperpolarized HDO with the waiting sample. Most of the protein is thus not visible due to deuteration of the amide protons (and consequently, in most cases we could not detect any protein signals for the samples measured here after their return to thermal equilibrium.) The required initial protein concentrations are thus comparable to those used in standard experiments. Until dilution during dissolution can be reduced, this will remain a primary limitation of the method.

For exchanging sites however, the loss in signal intensity due to limited proton availability in the solvent fraction is compensated due to the large enhancements (often greater than 500-fold) carried by the incoming hyperpolarized HDO, making them selectively visible, and doing so very rapidly when exchange is favorable. A key benefit of the reduced acquisition times demonstrated here should thus be significantly improved time-resolution for monitoring of rapid processes involving growth or evolution of small populations of transient species which can be made visible and resolvable using dissolution DNP. While it has been shown here that both folded and intrinsically disordered proteins are amenable to the method, IDPs in particular suffer from wellknown resolution limitations when studied by ${ }^{1} \mathrm{H}^{15} \mathrm{~N} 2 \mathrm{D}$ correlation methods. Hyperpolarized ${ }^{13} \mathrm{C}$-detected experiments can reduce these penalties, by providing improved spectral dispersion and reducing the broadening effects of rapid solvent exchange. Thus, while the $\mathrm{H}^{\mathrm{N}}-\mathrm{CON}$ may not be particularly informative for samples like ubiquitin, which was used here to provide a proof of concept, for systems like OPN where extensive broadening renders attempts to resolve hyperpolarized ${ }^{1} \mathrm{H} /{ }^{15} \mathrm{~N}$ correlations difficult or even impossible, the experiment should prove valuable, as it can provide useful site-specific information when alternative D-DNP-enhanced experiments would otherwise be unavailable. Furthermore, the extensive solvent exposure of IDPs facilitates good water-based enhancements, while preserving access to site-specific information about exchange. These 
combined resolution and sensitivity benefits are made available in hyperpolarized 2D H ${ }^{\mathrm{N}}$-CON NMR experiments.

Hyperpolarized 3D experiments were also shown here to be feasible via the use of NUS, which made 3D acquisitions compatible with the limited lifetime of hyperpolarized HDO. The power of the resulting method was demonstrated by its ability to discern residues involved in the binding undergone by a relatively large IDP like osteopontin. It also permitted the detection of small protein populations whose characterization would have been challenging or impossible by other NMR methods.

We expect the proposed experiments to be particularly useful for the study of phenomena such as post-translational modifications (Bah and Forman-Kay 2016), as such protein modification sites are frequently solvent-exposed and hence particularly well suited for investigation by water-derived hyperpolarized experiments.

\section{Material and methods}

\section{Dissolution and polarization conditions}

For the hyperpolarized $\mathrm{H}^{\mathrm{N}}-\mathrm{CON}$ experiments, $150 \mu \mathrm{L}$ of a $10 \mathrm{mM}$ solution of 4-amino-TEMPO in an 85:15 v/v mixture of water and glycerol was vitrified in liquid helium. The glassy sample was positively hyperpolarized for $\sim 3 \mathrm{~h}$ at $\sim 1.16 \mathrm{~K}$ in a magnetic field of $3.35 \mathrm{~T}$ by partial saturation of the EPR spectrum at $94.195 \mathrm{GHz}$ (Oxford HyperSense ${ }^{\mathrm{TM}}$, Weizmann Institute). Dissolution was achieved with a burst of $2.8 \mathrm{~mL}$ deuterated $20 \mathrm{mM}$ phosphate buffered saline (PBS) at $180{ }^{\circ} \mathrm{C}$ under $0.9 \mathrm{MPa}$. The sample was subsequently propelled to the nearby detection NMR spectrometer operating at $14.1 \mathrm{~T}\left(600 \mathrm{MHz}{ }^{1} \mathrm{H} \mathrm{NMR}\right)$ and $37{ }^{\circ} \mathrm{C}$ within $1.5 \mathrm{~s}$ with $\mathrm{He}$ gas (Olsen et al. 2016), where it was mixed with $150 \mu \mathrm{L}$ of target protein solution. Transfer and injection of hyperpolarized HDO was performed using an automated high-pressure injection system optimized to deliver reproducible volumes of solvent to the waiting protein solution while suppressing bubble and foam formation during mixing (Szekely et al. 2018; Olsen et al. 2016; Katsikis et al. 2015). NMR experiments were then triggered after a $3 \mathrm{~s}$ delay for mixing and settling.

For the hyperpolarized HNCO experiments, $180 \mu \mathrm{L}$ of a $15 \mathrm{mM}$ TEMPOL solution in an 85:15 v/v mixture of water and glycerol was vitrified in liquid helium. The glassy sample was positively hyperpolarized at $1.2 \mathrm{~K}$ in a magnetic field of $6.7 \mathrm{~T}$ by partial saturation of the EPR spectrum at $187.7 \mathrm{GHz}$ (Bruker Biospin, ENS Paris). Under these conditions, the hyperpolarization build-up rates were found to be $R_{\text {build-up }}=(4.8 \pm 0.1) \times 10^{-5} \mathrm{~s}^{-1}$, hence hyperpolarization buildup times of $3 \mathrm{~h}$ were used. Dissolution was achieved with a burst of $5 \mathrm{~mL} \mathrm{D}_{2} \mathrm{O}$ at $180{ }^{\circ} \mathrm{C}$ under
$1.05 \mathrm{MPa}$. The sample was subsequently propelled to a detection NMR spectrometer operating at $18.8 \mathrm{~T}(800 \mathrm{MHz}$ for ${ }^{1} \mathrm{H}$ ) and $37^{\circ} \mathrm{C}$ within $1.3 \mathrm{~s}$ with $\mathrm{He}$ gas under $0.7 \mathrm{MPa}$ through a "magnetic tunnel" (Milani et al. 2015) maintaining a constant magnetic field of $0.9 \mathrm{~T}$ over a distance of approximately $4 \mathrm{~m}$. The delay for mixing with the target protein solution $(150 \mu \mathrm{L})$ and settling of turbulence prior to initiation of the NMR experiments was again $3 \mathrm{~s}$.

\section{NMR spectroscopy}

${ }^{1} \mathrm{H}$ and ${ }^{13} \mathrm{C}$ chemical shifts were referenced to DSS, ${ }^{15} \mathrm{~N}$ chemical shifts were referenced according to Markley et al. (1998).

\section{$\mathrm{H}^{\mathrm{N}}$-CON experiments}

The $\mathrm{H}^{\mathrm{N}}$-CON experiments were implemented as described by Felli and co-workers (Gil et al. 2013). All $\mathrm{H}^{\mathrm{N}}$-CON spectra were recorded on a $14.1 \mathrm{~T}(600 \mathrm{MHz})$ Bruker Avance III ${ }^{\circledR}$ NMR spectrometer equipped with a 5 mm Prodigy ${ }^{\circledR}$ cryoprobe. Bandwidths and offsets of shaped pulses were optimized empirically to maximize excitation of amide protons while minimizing unwanted depletion of the hyperpolarized HDO pool, as well as radiation damping effects that would result if the hyperpolarized water resonance were excited. As the HDO resonance in hyperpolarized water experiments is typically quite broad, the bandwidths of the proton excitation and refocusing pulses are more tightly constrained than in experiments at thermal equilibrium. In our hands, a good compromise for avoiding excitation of the water resonance while still exciting the target $\mathrm{H}^{\mathrm{N}}$ amide protons of interest was obtained by applying the proton excitation and refocusing pulses at $9.5-10.5 \mathrm{ppm}$ while increasing their bandwidths to $\sim 6 \mathrm{ppm}$. Selective ${ }^{1} \mathrm{H}$ excitation and refocusing were achieved using PC9 (Kupce and Freeman 1994) (bandwidth 6.25 ppm, duration $2001 \mu \mathrm{s}$ ), and REBURP (Geen and Freeman 1991) (bandwidths 6.0-6.05 ppm, durations $1600-1614 \mu \mathrm{s})$ pulses, respectively. Selective proton pulses were centered at $9.75 \mathrm{ppm}(\mathrm{OPN})$ or $10.5 \mathrm{ppm}$ (ubiquitin).

Selective ${ }^{13} \mathrm{C}$ excitation and refocusing were achieved using Q5 (bandwidth $136 \mathrm{ppm}$, duration $300 \mu \mathrm{s}$ ), and Q3 (bandwidth $115 \mathrm{ppm}$, duration $199 \mu \mathrm{s}$ ) pulses (Emsley et al. 1990). Selective ${ }^{13} \mathrm{C}$ pulses were centered at 173 and $54 \mathrm{ppm}$ for $\mathrm{CO}$ and $\mathrm{C} \alpha$, respectively. IPAP acquisition was used to suppress $\mathrm{CO}-\mathrm{C} \alpha$ couplings during acquisition. Non-selective ${ }^{15} \mathrm{~N} 90^{\circ}$ pulse durations were $31.5 \mu$ s (ubiquitin) and $32.9 \mu \mathrm{s}$ (OPN).

The spectral widths used were 19.9 and $26.3 \mathrm{ppm}$, respectively, for ${ }^{13} \mathrm{C}$ and ${ }^{15} \mathrm{~N}$ for ubiquitin, and 30.0 and $26.3 \mathrm{ppm}$ for OPN. 
For ubiquitin, the acquisition time in the direct dimension was $85 \mathrm{~ms}$, the recycle delay was $350 \mathrm{~ms}$, and the total time per transient was $537 \mathrm{~ms}$. A total of 64 FIDs (in-phase plus anti-phase) were collected, which were combined to give 16 complex increments. Two scans were collected per increment, to give a total experimental duration of $69 \mathrm{~s}$.

For OPN, the acquisition time in the direct dimension was $56 \mathrm{~ms}$, the recycle delay was $50 \mathrm{~ms}$, and the total time per transient $210 \mathrm{~ms}$. A total of 128 FIDs (in-phase plus anti-phase) were collected, which were combined to give 32 complex increments. Two scans were again collected per increment, to give a total experimental duration of $55 \mathrm{~s}$.

Reference $\mathrm{H}^{\mathrm{N}}-\mathrm{CON}$ spectra for ubiquitin and unbound OPN were collected at thermal equilibrium using experimental parameters matched to the corresponding hyperpolarized experiments, but with longer experimental durations, and using $2 \mathrm{mM}$ samples in $90 \%$ protonated PBS buffer. For the ubiquitin reference, the number of scans was again 2 , the recycle delay was increased to $1 \mathrm{~s}$, and the number of $\mathrm{t}_{1}$ increments collected was increased to 80 (20 complex $\mathrm{t}_{1}$ increments), to give a total experimental duration of $3 \mathrm{~min}$ $6 \mathrm{~s}$. For OPN, the number of scans was increased to 16 , the recycle delay was increased to $300 \mathrm{~ms}$, and the number of $t_{1}$ increments collected was doubled to 256 (64 complex $t_{1}$ increments), to give a total experimental duration of $32 \mathrm{~min}$.

\section{HNCO experiments}

${ }^{1} \mathrm{H},{ }^{13} \mathrm{C},{ }^{15} \mathrm{~N}$ BEST HNCO spectra were recorded on a wide-bore 18.8 T Bruker Avance III HD NMR spectrometer equipped with a TXI probe, using the Bruker library pulse sequence 'b_hncogp3d' (modified to generate shaped ${ }^{1} \mathrm{H}$ pulses directly from input values of offsets, flip-angles, and bandwidths) (Lescop et al. 2007). As for the $\mathrm{H}^{\mathrm{N}}-\mathrm{CON}$ experiments, shaped ${ }^{1} \mathrm{H}$ pulse bandwidth/offset combinations were again optimized empirically.

Selective ${ }^{1} \mathrm{H}$ pulses were PC9 (bandwidth $4.17 \mathrm{ppm}$, duration $2251 \mu \mathrm{s}$ ), REBURP (bandwidth $4.85 \mathrm{ppm}$, duration $1498 \mu \mathrm{s}), \mathrm{EBURP} 2$ and EBURP2tr (bandwidths $4.3 \mathrm{ppm}$, durations $1439 \mu \mathrm{s})$, and BIP720, 50, $20.1(150 \mu \mathrm{s})$ (Smith et al. 2001). Except for the BIP pulse (set on resonance at $4.7 \mathrm{ppm}$ ), all ${ }^{1} \mathrm{H}$ selective pulses were centered at $10.5 \mathrm{ppm}$ (ubiquitin and OPN) or 9.9 ppm (OPN-heparin).

Selective ${ }^{13} \mathrm{C}$ pulses were $\mathrm{G} 4$ and G4tr (bandwidth $125 \mathrm{ppm}$, duration $308 \mu \mathrm{s}$ ), and Q3 (bandwidth $82 \mathrm{ppm}$, duration $210 \mu \mathrm{s}$ ) (Emsley et al. 1990). Selective ${ }^{13} \mathrm{C}$ pulses were centered at $173 \mathrm{ppm}$ for $\mathrm{CO}$, or $53.2 \mathrm{ppm}$ for $\mathrm{C} \alpha$. Nonselective ${ }^{15} \mathrm{~N} 90^{\circ}$ pulse durations were $39.75 \mu \mathrm{s}$.

Spectral widths acquired were $13.95 \mathrm{ppm}$ for ${ }^{1} \mathrm{H}$, $35.0 \mathrm{ppm}$ for ${ }^{15} \mathrm{~N}$, and $14.0 \mathrm{ppm}$ for ${ }^{13} \mathrm{C}$. Carrier frequencies were $4.7 \mathrm{ppm}\left({ }^{1} \mathrm{H}\right), 173.5 \mathrm{ppm}\left({ }^{13} \mathrm{C}\right)$ and $117 \mathrm{ppm}\left({ }^{15} \mathrm{~N}\right)$. Poisson-gap NUS at $9.4 \%$ sampling was used, with sampling schedules generated using the HMSist 'nusPGSv3' AU macro (Hyberts et al. 2012). Twenty-four $\left\{\Delta t_{1}, \Delta t_{2}\right\}$ increment pairs were collected, to represent the $32 \times 8$ complex pairs $(\mathrm{TD}=512 \times 64 \times 16)$ of the corresponding fully-sampled HNCO experiment (or $512 \times 32 \times 32$, and 24 increment pairs, for the OPN + heparin sample). The acquisition time in the direct dimension was $23 \mathrm{~ms}$, the recycle delay $600 \mathrm{~ms}$ (500 $\mathrm{ms}$ for OPN + heparin), and the total time per transient $664 \mathrm{~ms}$, to give a total experimental time of $75 \mathrm{~s}(65 \mathrm{~s}$ for OPN + heparin). The full 3D spectra were reconstructed using the hmsIST (Hyberts et al. 2012) add-on in NMRPipe (Delaglio 1995) and analyzed with the Sparky (Goddard and Kneller 2008) program package.

Reference HSQC spectra were collected at thermal equilibrium using the standard Bruker sequence 'fhsqcf3gpph' (Mori et al. 1995). Signals were averaged over 16 scans, and 256 real and 256 imaginary $t_{1}$ points $\left(\Delta t_{1}=280 \mu \mathrm{s}\right)$ were collected, with a recovery delay of $1 \mathrm{~s}$. The ${ }^{1} \mathrm{H}$ and ${ }^{15} \mathrm{~N}$ $90^{\circ}$ pulse durations were 9.7 and $39.7 \mu$ s, respectively. To avoid extended signal averaging which would be required for samples in 96-98\% deuterated buffers, the HSQC reference spectra were acquired using fresh samples of the same proteins prepared in a $90 \%$ protonated $\mathrm{PBS}$ buffer, at $\mathrm{pH} 7.4$ and $37^{\circ} \mathrm{C}$.

\section{Protein sample preparation}

Uniformly ${ }^{15} \mathrm{~N}$ and ${ }^{13} \mathrm{C}$ enriched ubiquitin and OPN samples were prepared as described (Kadeřávek et al. 2018; Platzer et al. 2011). Before dilution by hyperpolarized HDO, protein concentrations were $2 \mathrm{mM}$ in all cases.

Acknowledgements Open access funding provided by University of Vienna. Bruker BioSpin provided a prototype of a D-DNP polarizer to ENS in Paris. This work was supported by the French CNRS, the ERC (contracts 'dilute para water', Grant Agreement 339754, 'HYPROTIN', Grant Agreement 801936 and '2F4BIODYN', Grant Agreement 279519), by the CNRS infrastructure network TGIR-RMN-THC (FR 3050), Equipex grant 'Paris-en-Résonance' (Contract ANR-10EQPX-09), Israel Science Foundation Grant 965/18, the Kimmel Institute of Magnetic Resonance (Weizmann Institute), and the generosity of the Perlman Family Foundation. We are grateful to Kobi Zibzener for his help throughout the project.

Open Access This article is licensed under a Creative Commons Attribution 4.0 International License, which permits use, sharing, adaptation, distribution and reproduction in any medium or format, as long as you give appropriate credit to the original author(s) and the source, provide a link to the Creative Commons licence, and indicate if changes were made. The images or other third party material in this article are included in the article's Creative Commons licence, unless indicated otherwise in a credit line to the material. If material is not included in the article's Creative Commons licence and your intended use is not permitted by statutory regulation or exceeds the permitted use, you will need to obtain permission directly from the copyright holder. To view a copy of this licence, visit http://creativecommons.org/licenses/by/4.0/. 


\section{References}

Bah A, Forman-Kay JD (2016) Modulation of intrinsically disordered protein function by post-translational modifications. J Biol Chem 291:6696-6705

Bertini I, Felli IC, Gonnelli L, Kumar MVV, Pierattelli R $(2011){ }^{13} \mathrm{C}$ direct-detection biomolecular NMR spectroscopy in living cells. Angew Chem Int Ed Engl 50:2339-2341

Clemente $\mathrm{N}$ et al (2016) Osteopontin bridging innate and adaptive immunity in autoimmune diseases. J Immunol Res 2016:7675437

Delaglio F et al (1995) NMRpipe-a multidimensional spectral processing system based on Unix pipes. J Biomol NMR 6:277-293

Doll A, Bordignon E, Joseph B, Tschaggelar R, Jeschke G (2012) Liquid state DNP for water accessibility measurements on spinlabeled membrane proteins at physiological temperatures. J Magn Reson 222:34-43

Emsley L, Bodenhausen G (1990) Gaussian pulse cascades: new analytical functions for rectangular selective inversion and in-phase excitation in NMR. Chem Phys Lett 165(2):469-475

Geen H (1991) Freeman R (1991) Band-selective radiofrequency pulses. J Magn Reson 93:93-141

Gil S et al (2013) NMR spectroscopic studies of intrinsically disordered proteins at near-physiological conditions. Angew Chem Int Ed 52:11808-11812

Goddard TD, Kneller DG (2008) SPARKY. University of California, San Francisco

Hamilton KS, Ellison MJ, Shaw GS (2000) Identification of the ubiquitin interfacial residues in a ubiquitin-E2 covalent complex. J Biomol NMR 18:319-327

Hyberts SG, Milbradt AG, Wagner AB, Arthanari H, Wagner G (2012) Application of iterative soft thresholding for fast reconstruction of NMR data non-uniformly sampled with multidimensional Poisson Gap scheduling. J Biomol NMR 52:315-327

Kadeřávek P, Ferrage F, Bodenhausen G, Kurzbach D (2018) Highresolution NMR of folded proteins in hyperpolarized physiological solvents. Chem Eur J 24:13418-13423

Katsikis S, Marin-Montesinos I, Pons M, Ludwig C, Gunther UL (2015) Improved stability and spectral quality in ex situ dissolution DNP using an improved transfer device. Appl Magn Reson 46:723-729

Kazimierczuk K, Zawadzka A, Kozminski W (2009) Narrow peaks and high dimensionalities: exploiting the advantages of random sampling. J Magn Reson 197:219-228

Kazimierczuk K, Zawadzka-Kazimierczuk A, Kozminski W (2010) Non-uniform frequency domain for optimal exploitation of nonuniform sampling. J Magn Reson 205:286-292

Kim J, Liu M, Hilty C (2017) Modeling of polarization transfer kinetics in protein hydration using hyperpolarized water. J Phys Chem B 121:6492-6498

Kupce E, Freeman R (1994) Wideband excitation with polychromatic pulses. J Magn Reson A 108:268-273

Kurzbach D et al (2013) Cooperative unfolding of compact conformations of the intrinsically disordered protein osteopontin. Biochemistry $52: 5167-5175$

Kurzbach D et al (2017) Investigation of intrinsically disordered proteins through exchange with hyperpolarized water. Angew Chem Int Ed Engl 56:389-392

Lescop E, Schanda P, Brutscher B (2007) A set of BEST triple-resonance experiments for time-optimized protein resonance assignment. J Magn Reson 187:163-169

Lipso KW, Bowen S, Rybalko O, Ardenkjaer-Larsen JH (2017) Large dose hyperpolarized water with dissolution-DNP at high magnetic field. J Magn Reson 274:65-72
Markley JL et al (1998) Recommendations for the presentation of NMR structures of proteins and nucleic acids. IUPAC-IUBMB-IUPAB Inter-Union Task Group on the standardization of data bases of protein and nucleic acid structures determined by NMR spectroscopy. J Biomol NMR 12:1-23

Mateos B, Konrat R, Pierattelli R, Felli IC (2019) NMR characterization of long-range contacts in intrinsically disordered proteins from paramagnetic relaxation enhancement in ${ }^{13} \mathrm{C}$ direct-detection experiments. ChemBioChem 20:335-339

Mayzel M, Rosenlow J, Isaksson L, Orekhov VY (2014) Time-resolved multidimensional NMR with non-uniform sampling. J Biomol NMR 58:129-139

Milani J et al (2015) A magnetic tunnel to shelter hyperpolarized fluids. Rev Sci Instrum 86:024101

Modig K, Liepinsh E, Otting G, Halle B (2004) Dynamics of protein and peptide hydration. J Am Chem Soc 126:102-114

Mori S, Abeygunawardana C, Johnson MO, Zijl PCV (1995) Improved sensitivity of HSQC spectra of exchanging protons at short interscan delays using a new Fast HSQC (FHSQC) detection scheme that avoids water saturation. J Magn Reson B 108:94-98

Nucci NV, Pometun MS, Wand AJ (2011) Site-resolved measurement of water-protein interactions by solution NMR. Nat Struct Mol Biol 18:245-249

Olsen G, Markhasin E, Szekely O, Bretschneider C, Frydman L (2016) Optimizing water hyperpolarization and dissolution for sensitivity-enhanced 2D biomolecular NMR. J Magn Reson 264:49-58

Otting G (1997) NMR studies of water bound to biological molecules. Prog Nucl Magn Reson Spectrosc 31:259-285

Platzer G et al (2011) The metastasis-associated extracellular matrix protein osteopontin forms transient structure in ligand interaction sites. Biochemistry 50:6113-6124

Ragavan M, Iconaru LI, Park CG, Kriwacki RW, Hilty C (2017) Realtime analysis of folding upon binding of a disordered protein by using dissolution DNP NMR spectroscopy. Angew Chem Int Ed Engl 56:7070-7073

Reichheld SE, Muiznieks LD, Keeley FW, Sharpe S (2017) Direct observation of structure and dynamics during phase separation of an elastomeric protein. Proc Natl Acad Sci U S A 114(22):E4408-E4415

Rodrigues LR, Teixeira JA, Schmitt FL, Paulsson M, LindmarkMansson H (2007) The role of osteopontin in tumor progression and metastasis in breast cancer. Cancer Epidemiol Biomark Prev 16:1087-1097

Schanda P, Kupce E, Brutscher B (2005) SOFAST-HMQC experiments for recording two-dimensional heteronuclear correlation spectra of proteins within a few seconds. J Biomol NMR 33:199-211

Smith MA, Hu H, Shaka AJ (2001) Improved broadband inversion performance for NMR in liquids. J Magn Reson 151:269-283

Szekely O, Olsen GL, Felli IC, Frydman L (2018) High-resolution 2D NMR of disordered proteins enhanced by hyperpolarized water. Anal Chem 90(10):6169-6177

Theillet FX et al (2016) Structural disorder of monomeric alpha-synuclein persists in mammalian cells. Nature 530:45-50

Viennet $\mathrm{T}$ et al (2016) Selective protein hyperpolarization in cell lysates using targeted dynamic nuclear polarization. Angew Chem Int Ed Engl 55:10746-10750

Vijay-Kumar S, Bugg C, Cook WJ (1987) Structure of ubiquitin refined at 1.8 A resolution. J Mol Biol 194:531-544

Wang Y, Hilty C (2019) Determination of ligand binding epitope structures using polarization transfer from hyperpolarized ligands. J Med Chem 62:2419-2427 
Yuwen T, Skrynnikov NR (2014) CP-HISQC: a better version of HSQC experiment for intrinsically disordered proteins under physiological conditions. J Biomol NMR 58:175-192

Zawadzka-Kazimierczuk A, Kozminski W, Sanderova H, Krasny L (2012) High dimensional and high resolution pulse sequences for backbone resonance assignment of intrinsically disordered proteins. J Biomol NMR 52:329-337
Publisher's Note Springer Nature remains neutral with regard to jurisdictional claims in published maps and institutional affiliations. 